\title{
Generalization to novel views from view combination
}

\author{
KAVITHA SRINIVAS and JOHN SCHWOEBEL \\ Boston College, Chestnut Hill, Massachusetts
}

\begin{abstract}
Four experiments examined whether or not exposure to two views (A and B) of a novel object improves generalization to a third view (C) through view combination on tasks that required symmetry or recognition memory decisions. The results of Experiment 1 indicated that exposure to either View A or View B alone produced little or no generalization to View $\mathrm{C}$ on either task. The results of Experiment 2 indicated that exposure to both View $A$ and View B did improve generalization to View C, but only for symmetrical objects. Experiment 3 replicated this generalization advantage for symmetrical but not asymmetrical objects, when objects were well learned at study. The results of Experiment 4 showed that Views A and B did not have to be presented consecutively to facilitate responses to View C. Together, the pattern of results suggests that generalization to novel views does occur through view combination of temporally separated views, but it is more likely to be observed with symmetrical objects.
\end{abstract}

Adverse effects of rotating novel and familiar objects in depth between study and test have been observed on several different memory tasks, including recognition, naming, left-right decisions, and object-nonobject decisions (Bartram, 1974; Bulthoff \& Edelman, 1990; Bulthoff, Edelman, \& Sklar, 1991; Edelman \& Bulthoff, 1992; Humphrey \& Khan, 1992; Rock \& DiVita, 1987; Rock, DiVita, \& Barbeito, 1981; Srinivas, 1993, 1995; Tarr, 1995; Tarr, Hayward, Gauthier, \& Williams, 1994). These findings suggest that object representations specify shape from a particular experienced view.

The central problem examined in this paper is whether or not generalization to a novel view of an object can be improved through a process called view combination. Bulthoff and Edelman (1992) suggested that generalization to novel views may be improved by prior experience with more than one view of an object, through a process of linear interpolation of the previously learned views. To provide evidence for this suggestion, Bulthoff and Edelman (1992) examined recognition memory for wireframe objects following exposure to two studied views of the object that were separated by $75^{\circ}$ in depth. Each studied view was actually a set of two-dimensional (2-D) views that showed the object rotating by $15^{\circ}$ about the view to create the illusion of movement in depth. Following train-

This research was supported by a Distinguished Junior Faculty Award from Boston College. Part of this work was presented at the 1994 meeting of the Psychonomic Society in St. Louis. The authors would like to thank Joni Hemond, Marina latropoulous, Margaret Machado, Jamie Norris, Michael Rennick, Padma Sankaran, and Amanda Steege for their help in testing participants, and Randolph Easton, Jeanne Sholl, and Suparna Rajaram for comments on an earlier version of this manuscript. Correspondence should be addressed to K. Srinivas, Department of Psychology, Boston College, Chestnut Hill, MA 02167-3807 (e-mail: kavitha.srinivas@bc.edu).

-Accepted by previous editor, Geoffrey R. Loftus ing with these two studied views, subjects were tested on unseen views that were either (1) "interpolated" views between the two studied views (i.e., between $0^{\circ}-75^{\circ}$, assuming that the two studied views were at $0^{\circ}$ and $75^{\circ}$, respectively) or (2) "extrapolated" views that were views beyond the two studied views (i.e., $75^{\circ}-360^{\circ}$ ). The interesting result for the purposes of the present research is that Bulthoff and Edelman (1992) found substantial generalization to interpolated views, and less generalization to extrapolated views of wire-frame objects, with recognition memory accuracy as the measure of generalization. For amoeba-like objects, generalization occurred for both interpolated and extrapolated views. These results have recently been replicated in a naming task for interpolated views of novel objects composed of several "bricks" (Tarr, 1995). The measure of generalization in this study was a decrease in naming times for interpolated views of learned views relative to naming times for extrapolated views.

The Bulthoff and Edelman (1992) and Tarr (1995) experiments demonstrated that significant generalization to a novel view occurs after experience with more than one view of an object, for both recognition and naming tasks. In the present research, we extend their findings to examine the nature of this generalization process. Specifically, we outline three key issues regarding the process of view combination below, because they provide the rationale for the present experiments.

First, we examined whether view combination produces generalization to novel views for the "basic-level" identification of objects (i.e., identification of objects with dissimilar perceptual structures, such as dogs and horses). Both Bulthoff and Edelman (1992) and Tarr (1995) demonstrated view combination when the target objects in the Experiment were perceptually similar. Hence, one might argue that view combination applies only to the discrimination of highly similar object structures (e.g., the discrimination of a Honda from a Toyota, or "within- 
category" discrimination) rather than to the discrimination of objects with dissimilar structures (i.e., basic-level identification). In the present experiments, we employed objects with dissimilar structures and distinct parts to examine whether view combination also plays a role in basiclevel identification.

Second, we explored whether view combination occurs only upon exposure to three-dimensional (3-D) information about the object. In Bulthoff and Edelman's (1992) study, participants experienced apparent motion for each set of study views, allowing access to depth information through the kinetic depth effect. Similarly, in Tarr's (1995) experiment, training consisted of exposure to different views of an object, as well as exposure to a 3-D model of the object in a particular view. In the present study, two 2 -D views of each object were presented nonconsecutively at study in order to examine whether or not improved generalization to novel views is dependent on access to 3-D object information.

Third, we explored whether view combination provides generalization to novel views over and above any generalization that may be obtained from the views presented in isolation. In prior studies, this control was not necessary because of the extreme specificity in viewpoint that has been repeatedly demonstrated for highly similar objects (e.g., Rock \& DiVita, 1987; Tarr, 1995). However, this control is essential for perceptually dissimilar objects, because some generalization to novel views could occur from exposure to the views alone, rather than due to view combination (Biederman \& Gerhardstein, 1993; but see Srinivas, 1995; Tarr et al., 1994).

To examine these issues, we measured generalization to a novel view (C) from exposure to two views (A and $B$ ) for symmetrical and asymmetrical objects. Generalization was measured on a recognition memory task and a symmetry decision task at test to allow a conceptual replication of prior findings. We adopted a symmetry decision task instead of the naming task used in Tarr's (1995) study because it eliminated the need for extensive training at study and because it allowed us to test a larger set of objects than that used by Tarr. Our first experiment was a control experiment that was designed to examine whether exposure to Views A and B in isolation provided any generalization to View C. In Experiment 2, we examined whether exposure to Views $\mathrm{A}$ and $\mathrm{B}$ together provided generalization to View C. To anticipate our results, we demonstrated generalization in Experiment 2 with symmetrical but not asymmetrical objects. In Experiment 3, we attempted a replication of Experiment 2, under conditions where the symmetrical and asymmetrical objects were well learned. In Experiment 4, we examined whether Views A and B had to be presented contiguously to produce generalization to View $\mathrm{C}$.

\section{EXPERIMENT 1}

Experiment 1 was designed to examine the degree of generalization produced by two views (Views $\mathrm{A}$ or $\mathrm{B}$ ) to a given third view (View $C$ ). The stimulus materials and design were an extension of Srinivas's (1995) Experiment 3 , in which she found view sensitivity when novel objects were rotated by $80^{\circ}$ at test on a left-right decision task and a recognition memory task.

\section{Method}

Participants. Ninety-six Boston College undergraduates participated in order to partially fulfill a course requirement.

Materials. Thirty-six symmetrical objects from Srinivas's (1995) Experiment 3 were used in this experiment. A new set of 36 asymmetrical objects was created because the symmetry decision task required the use of symmetrical and asymmetrical objects. Each of the 72 multipart objects used in this and other experiments reported here was composed of distinctively shaped wooden pieces. All objects had head and tail pieces, as well as arm pieces attached to a vertically elongated main part. For 36 of the objects, the arms were attached in order to form bilateral symmetry as in Srinivas (1995), whereas, in the remaining 36 objects, the position of the "arms" formed asymmetrical objects. Note that, for asymmetrical objects, the structure could not be described in terms of head, arm, or tail pieces; hence, the manipulation of arm pieces for these objects was arbitrary. Three views of each object were digitized under even illumination and with a uniform black background. The three views consisted of an arbitrary test view (View $C$ ) and $80^{\circ}$ (View A) and $110^{\circ}$ (View B) rotations from that test view. All three views had the same object parts visible in each view. For half of the objects, the test view was one in which the object was facing left, and the two rotated views were facing right, whereas the opposite was true for the other half of the objects. Note that View $\mathrm{C}$ was at least $80^{\circ}$ from Views $A$ and $B$; hence, the process by which generalization could occur to the test view from these two views was extrapolation rather than interpolation (in Bulthoff and Edelman's, 1992, terms). Each view was digitized as an 8 -bit $(256$ grays) $640 \times 480$ resolution picture, and it could be contained within a $320 \times 240$ pixel rectangle that was centered on the screen. (See Figure 1 for examples of Views C, A, and B for symmetrical objects, and see Figure 2 for examples of asymmetrical objects.) In the figures, Views C, A, and B are labeled $0^{\circ}, 80^{\circ}$, and $110^{\circ}$, respectively, to indicate the degree of rotation from the test view (which in the figures is the $0^{\circ}$ view). This terminology is adopted for the rest of the paper because it specifies the degree of rotation precisely.

Design. A 2 (test type: symmetry decision or recognition) $\times 2$ (study status: studied or nonstudied) $\times 3$ (study view: $0^{\circ}, 80^{\circ}$, or $110^{\circ}$ ) mixed factorial design was used. Test type was manipulated between subjects, whereas study status and study view were manipulated within subjects. Forty-eight participants were given the symmetry decision task, and an additional 48 participants were assigned to the recognition task. For each task, 36 objects were presented at study and 72 objects were presented at test. Of the 72 test objects, 12 were presented in exactly the same view at study and test $\left(0^{\circ}\right.$ condition), 12 were presented at study in a view that was rotated $80^{\circ}$ from the test view $\left(80^{\circ}\right.$ condition), 12 were presented at study in a view that was rotated $110^{\circ}$ from the test view $\left(110^{\circ}\right.$ condition $)$, and 36 were nonstudied objects. Note that, in all three conditions $\left(0^{\circ}\right.$, $80^{\circ}$, and $\left.110^{\circ}\right)$, the test view was always exactly the same $\left(0^{\circ}\right.$ view $)$. The manipulation of rotation occurred at study, so that the studied view either was identical to the test view or was an $80^{\circ}$ or $110^{\circ}$ rotation of the test view. This design therefore ensured that any differences observed at test for the three conditions could not be attributed to differences in canonicality of the $0^{\circ}, 80^{\circ}$, or $110^{\circ}$ views; rather, the differences could only be due to the viewpoint in which the object had been presented at study. Six study and test lists were used to achieve counterbalancing so that each item was presented in each condition equally often across subjects.

Procedure. The participants were tested in groups of 3 or less on three IBM-compatible computers. At study, all participants were given exactly the same instructions. After a brief description of bi- 


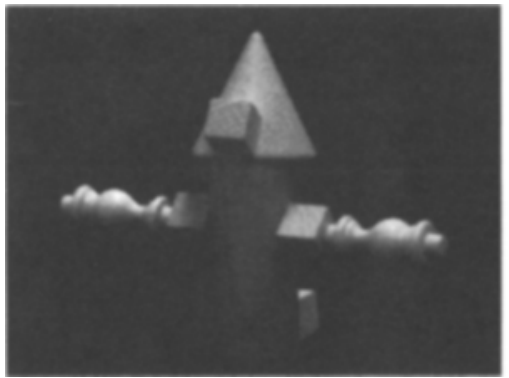

$0^{\circ}$ view

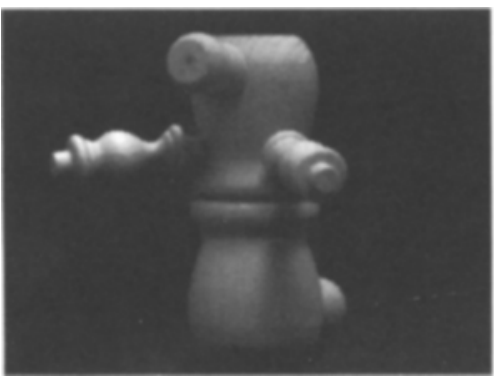

$0^{\circ}$ view

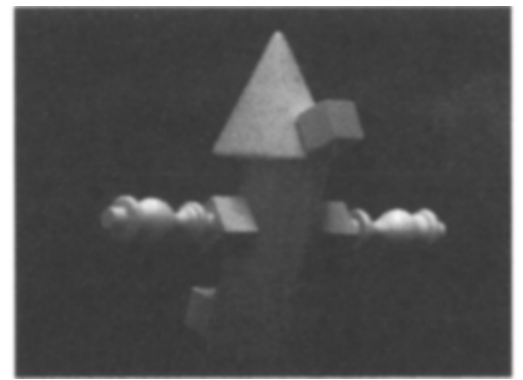

$80^{\circ}$ view

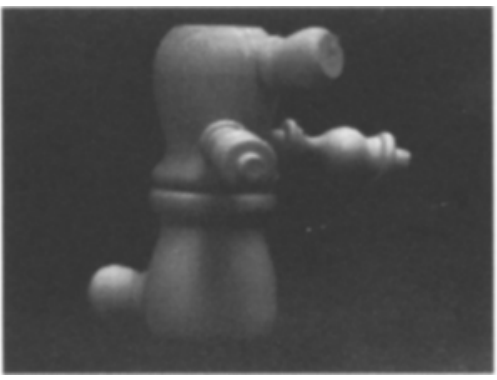

$80^{\circ}$ view

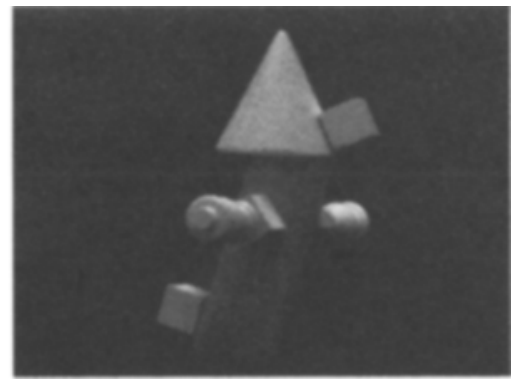

$110^{\circ}$ view

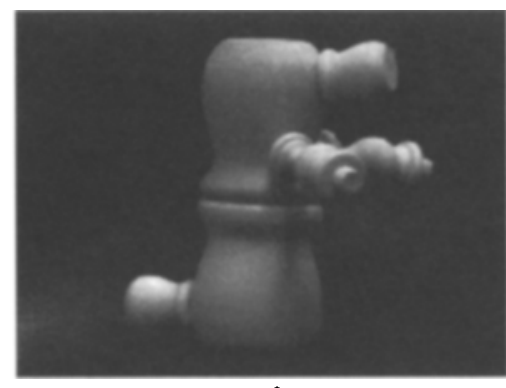

$110^{\circ}$ view

Figure 1. Examples of the $0^{\circ}, 80^{\circ}$, and $110^{\circ}$ views of symmetrical objects presented in Experiments 1-4.

lateral symmetry that involved demonstrations of symmetry in familiar objects (such as humans), the participants were told that they would see pictures of novel wooden objects for a maximum of $5 \mathrm{sec}$ each and were asked to press the green mouse button if an object was symmetrical and to press the red mouse button if an object was asymmetrical as quickly and as accurately as possible. The participants were also told to use the index and middle fingers of their preferred hand to make the responses. The first 10 objects presented were for practice and included the correct response (symmetrical or asymmetrical) on the screen. No mention was made of the later test phase in the instructions. After the study phase, the participants were engaged in a 5-min distractor task, which required them to solve anagrams, and then the test instructions were given.

For the symmetry decision task, the participants were again asked to make symmetry decisions for another set of novel objects. They were told that the experimenter was interested in the effects of practice on the task. Response mode and presentation times were exactly the same as at study. For the recognition task, the participants were asked to press the green mouse button if an object was seen during the study phase (studied) and to press the red mouse button if an object was not seen during the study phase (nonstudied) as quickly and accurately as possible. The participants were also told to press the green mouse button if an object was studied, regardless of any change in viewpoint from study to test. Stimuli were presented in a random order both at study and at test; this order was determined separately for each participant. A C program was used to control the random presentation of stimuli and to record latency and accuracy data.

\section{Results and Discussion}

For each experiment reported in this paper, the results for different tasks (symmetry decision or recognition) are presented in different sections because of procedural and baseline differences between the types of memory tests. Furthermore, separate analyses are reported for the responses to symmetrical and asymmetrical objects, because it is clear from earlier research that there are noticeable differences in memory for symmetrical and asymmetrical objects. Specifically, Kersteen-Tucker (1991) reported little or no facilitation (or priming) from the repetition of asymmetrical objects on a symmetry decision task, whereas symmetrical objects showed robust facilitation under the same conditions. Given these possible differences in memory for symmetrical and asymmetrical objects, we chose to analyze the data separately for the two types of objects.

In all experiments reported here, both latency and accuracy data were analyzed for the two tasks. However, accuracy rates were at ceiling on the symmetry decision task, and accuracy was close to $50 \%$ on most of the conditions in the recognition memory task, leading to inadequate statistical power in the latency analyses. Hence, latency was the primary dependent measure for the symmetry decision task, and a corrected recognition score (hits minus false alarms) was the primary measure for the recognition memory task. We analyzed accuracy for the symmetry decision tasks, and latency for the recognition task, and we failed to obtain any reliable differences in either measure. This analysis rules out the possibility of speed-accuracy tradeoffs. 


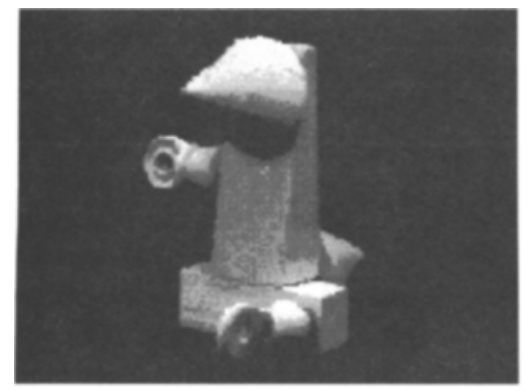

$0^{\circ}$ view

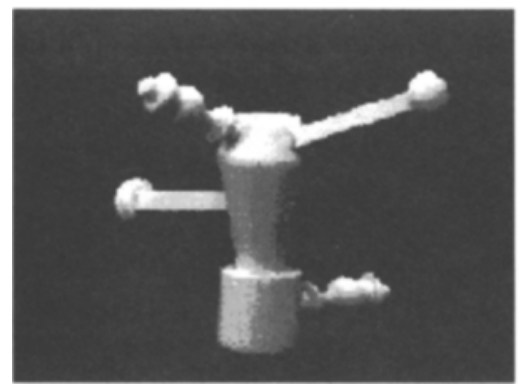

$0^{\circ}$ view

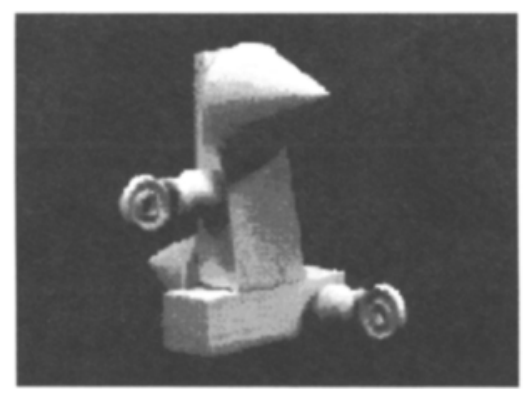

$80^{\circ}$ view

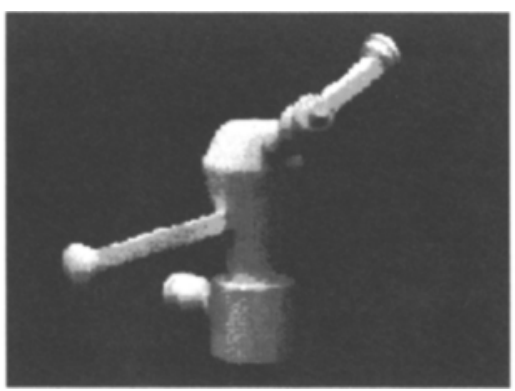

$80^{\circ}$ view

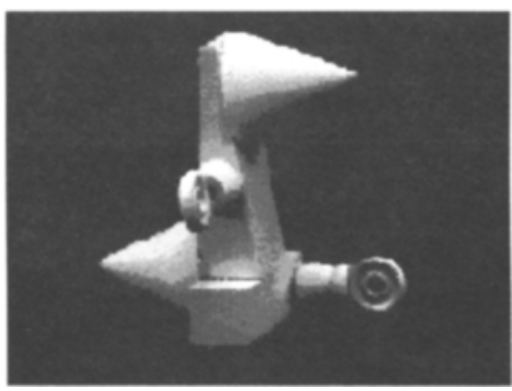

$110^{\circ}$ view

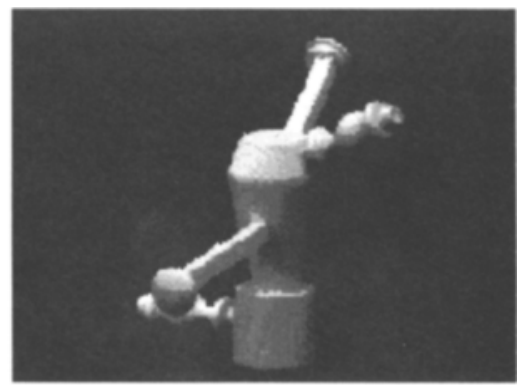

$110^{\circ}$ view

Figure 2. Examples of the $0^{\circ}, 80^{\circ}$, and $110^{\circ}$ views of asymmetrical objects presented in Experiments $1-4$.

Latency data in all experiments are reported only for correct responses because latencies for incorrect responses are not theoretically interesting for our purposes. Analyses by subject and by item variability were performed for all the results reported, but because the two analyses were consistent, unless otherwise stated, only the analyses by subjects are reported. The criterion for reporting results as statistically significant is the .05 level of significance, unless stated otherwise. The $95 \%$ confidence intervals shown on the bars of all figures were calculated using the within-subjects mean square error $\left(M S_{\mathrm{e}}\right)$ for the effect of study view for both tasks, because this analysis allowed for the most efficient comparison of the relative performance in the different rotation conditions (see Loftus \& Masson, 1994).

For the symmetry decision tasks, data from outlier participants with mean response times greater than 2 standard deviations ( $S D s$ ) from the grand mean for the correct studied items were deleted and replaced. The same procedure was used to identify outliers in the recognition tasks using the accuracy data. This procedure resulted in only 5 participants being replaced across all experiments, 2 of who were replaced in the symmetry decision task of Experiment 1 , and 3 who were replaced in the symmetry decision task of Experiment 3.

Symmetry decision task. Figure 3 represents latency (in milliseconds) for symmetrical and asymmetrical objects as a function of study condition $\left(0^{\circ}, 80^{\circ}\right.$, and $110^{\circ}$ conditions). Nonstudied base rates are indicated by horizontal lines in the figure. Memory as measured by priming on the task is therefore indicated by the difference between the top of the bar and the horizontal line.

We first examined priming as a function of exposure to the view itself at study $\left(0^{\circ}\right)$, or exposure to $80^{\circ}$ and $110^{\circ}$ views. For symmetrical objects, as indicated in Figure 3, statistically significant priming was obtained in the $0^{\circ}$ condition $\left[F(1,47)=9.15, M S_{\mathrm{e}}=127,382\right]$, but not in either the $80^{\circ}$ condition $(F \leq 1)$ or the $110^{\circ}$ condition $(F \leq$ 1). ${ }^{1}$ Response latency was shorter in the $0^{\circ}$ condition than was the combined latency in the $80^{\circ}$ and $110^{\circ}$ conditions $\left[F(1,47)=6.77, M S_{\mathrm{e}}=110,835\right]$, and there was no statistically significant difference between the $80^{\circ}$ and $110^{\circ}$ conditions $(F \leq 1)$. In other words, perceiving the $80^{\circ}$ or the $110^{\circ}$ view at study did not produce as much generalization to the $0^{\circ}$ test view as did exposure to the $0^{\circ}$ view. This result replicates previous findings by Srinivas (1995) and suggests view sensitivity for novel objects even when the same parts are visible in the different views. Interestingly, there is a suggestion in these results that no generalization is obtained to the test view from exposure to the $80^{\circ}$ or the $110^{\circ}$ view. As we see later in Experiment 3, limited generalization can be obtained from these views when objects are well learned at study.

As expected from Kersteen-Tucker's (1991) research, for asymmetrical objects, priming was not statistically significant. Planned comparisons indicated that priming 
Symmetry Decision Task

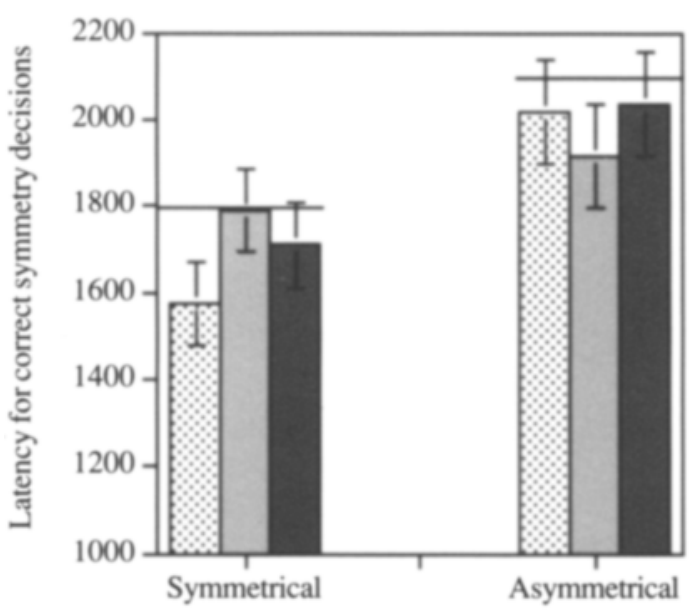

Recognition Task

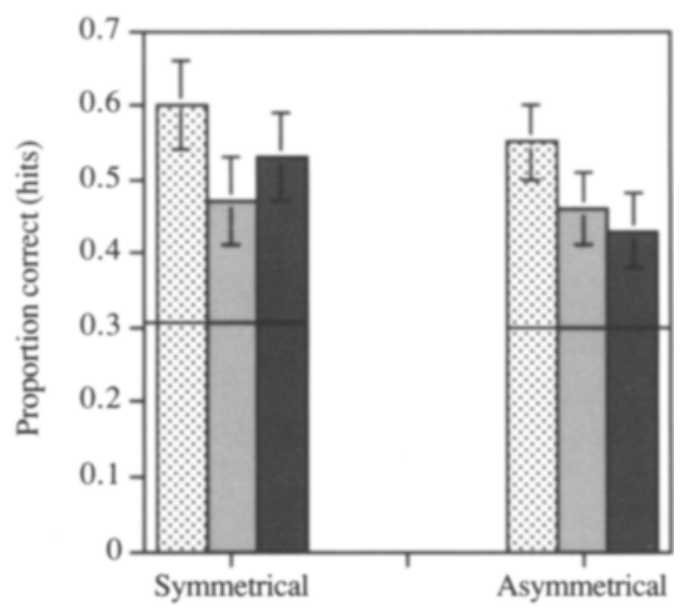

Views at Study

Figure 3. The latency and accuracy results for the symmetry-asymmetry task and the recognition memory task of Experiment 1 for the $0^{\circ}, 8^{\circ}$, and $110^{\circ}$ conditions. Error bars indicate $95 \%$ confidence intervals calculated using the within-subjects $M S_{\mathrm{e}}$ for the effect of study view for the symmetry decision and recognition memory tasks (see Loftus \& Masson, 1994). Horizontal lines on the graphs indicate false-alarm rates for the recognition memory task and nonstudied base rates for the the symmetry decision task.

was not statistically significant in either the $0^{\circ}$ condition $(F \leq 1)$ or the $110^{\circ}$ condition $(F \leq 1)$. Surprisingly, for the $80^{\circ}$ condition, a statistically significant effect was obtained by subject variability $\left[F(1,47)=12.99, M S_{\mathrm{e}}=60,752\right]$, but not by item variability $(F \leq 1)$. We should note that the surprising priming effect in the $80^{\circ}$ condition was statistically significant only by one analysis and was not replicated in Experiments 2, 3, and 4, and, hence, the result is not discussed further. As might be expected from the lack of priming for asymmetrical objects, planned comparisons revealed no statistically significant differences between the mean latencies for the different study conditions. There was no statistically significant difference between the $0^{\circ}$ condition and the combined $80^{\circ}$ and $110^{\circ}$ conditions $(F<1)$, and the difference between the $80^{\circ}$ and $110^{\circ}$ conditions was not consistently significant across the analyses by subject variability $\left[F(1,47)=2.89, M S_{\mathrm{e}}=\right.$ $127,014, p<.10]$ and item variability $(F<1)$.

Recognition memory task. Figure 3 provides accuracy data for the recognition responses to symmetrical and asymmetrical objects presented at test for the three study conditions $\left(0^{\circ}, 80^{\circ}\right.$, and $110^{\circ}$ conditions), along with falsealarm rates (horizontal lines). Again, memory as measured by hits - false alarms is indicated in the figure as a difference between the horizontal line and the top of the bar. As can be seen in Figure 3, recognition accuracy for the studied symmetrical objects was significantly above chance $(0)$ in the $0^{\circ}$ condition $[t(47)=6.65]$, the $80^{\circ}$ condition $[t(47)=3.73]$, and the $110^{\circ}$ condition $[t(47)=5.66]$. Planned comparisons between the mean accuracy for the three study conditions also revealed significantly higher accuracy for objects presented in the $0^{\circ}$ condition relative to the average of the $80^{\circ}$ and $110^{\circ}$ conditions $\left[F(1,47)=5.51, M S_{\mathrm{e}}=0.04\right]$ and revealed no statistically significant difference between the $80^{\circ}$ and $110^{\circ}$ conditions $\left[F(1,47)=2.31, M S_{\mathrm{e}}=0.04, p<.14\right]$. These results for symmetrical objects replicate earlier findings by Srinivas (1995), as well as the findings reported above for the symmetry decision task, and suggest that when subjects see one view of an object at study, recognition memory is sensitive to changes in viewpoint between study and test. The same pattern of findings was also observed for asymmetrical objects. For asymmetrical objects, recognition was significantly above chance in the $0^{\circ}$ condition $[t(47)=6.50]$, the $80^{\circ}$ condition $[t(47)=$ $4.79]$, and the $110^{\circ}$ condition $[t(47)=3.84]$. Planned comparisons also revealed an advantage for the $0^{\circ}$ condition relative to the average of the $80^{\circ}$ and $110^{\circ}$ conditions $\left[F(1,47)=9.36, M S_{\mathrm{e}}=0.03\right]$ and revealed no difference between the $80^{\circ}$ and $110^{\circ}$ conditions $(F<1)$. These findings suggest that recognition memory for asymmetrical objects is also best when exactly the same views of objects are seen at study and test.

In summary, for symmetrical objects, the greatest priming and best recognition memory performance were observed when the same views of objects were presented at study and test (i.e., $0^{\circ}$ condition). This finding is consistent with the idea that memory performance is based on view-sensitive representations of objects. However, an alternative explanation for the advantage observed in the 
$0^{\circ}$ condition is that the $0^{\circ}$ view is simply a better view for the perception of symmetry than is the $80^{\circ}$ or the $110^{\circ}$ view. To test this possibility, we conducted an analysis of response latency for the $0^{\circ}$ view versus the average latency for the $80^{\circ}$ and $110^{\circ}$ views of symmetrical objects presented in the study phase of the symmetry decision task. This analysis indicated no statistically significant difference $(F<1)$, suggesting that the $0^{\circ}$ view was not a "better" view for judging 3-D symmetry. Mean latencies for the $0^{\circ}, 80^{\circ}$, and $110^{\circ}$ views were $2,201,2,099$, and $2,373 \mathrm{msec}$, respectively. Similarly, no statistically significant difference between the latencies for the $0^{\circ}$ view and the combined $80^{\circ}$ and $110^{\circ}$ views was obtained in an analysis of latencies for the study phase of the recognition memory task [by subjects, $F(1,47)=2.37, M S_{\mathrm{e}}=198,741$, $p<.13$; by items, $F<1$ ]. Mean latencies for the $0^{\circ}, 80^{\circ}$, and $110^{\circ}$ views were $2,467,2,423$, and $2,770 \mathrm{msec}$, respectively. Thus, the advantage observed at test for the $0^{\circ}$ study condition cannot be interpreted as suggesting that the $0^{\circ}$ view is simply easier to process; instead, it appears to be due to the presentation of identical views at study and test. Therefore, these results suggest that memory for symmetrical objects, as measured by both symmetry decision and recognition tasks, is sensitive to changes in viewpoint.

For asymmetrical objects, surprisingly poor memory was observed in the symmetry decision task. This occurred even when the object was presented in exactly the same view at study and test. These results may indicate difficulties in the representation of asymmetrical objects or perhaps a bias of the type suggested by Ratcliff and McKoon (1995). According to the bias explanation, subjects tend to respond "symmetrical" to previously seen objects if they appear familiar, and, therefore, their correct responses to previously studied asymmetrical objects would be slowed down relative to nonstudied asymmetrical objects.

Importantly, the reduced generalization from either the $80^{\circ}$ view or the $110^{\circ}$ view at study to the $0^{\circ}$ view at test provides an important constraint on the interpretation of the results in Experiments 2-4. If improved performance is observed for the $0^{\circ}$ view after exposure to the $80^{\circ}$ view and the $110^{\circ}$ view, this facilitation cannot be attributed simply to generalization from either one of the two views.

\section{EXPERIMENT 2}

In Experiment 2, we explored whether or not displaying two consecutive views (the $80^{\circ}$ and $110^{\circ}$ views) of an object at study would improve generalization to the $0^{\circ}$ test view. We examined performance on the symmetry decision and recognition tasks as a function of three conditions: (1) In the $0^{\circ}-0^{\circ}$ condition, the $0^{\circ}$ view was presented twice consecutively at study, (2) in the $80^{\circ}-110^{\circ}$ condition, the $80^{\circ}$ and $110^{\circ}$ views were presented consecutively at study, and (3) in the $80^{\circ}-80^{\circ}$ condition, the $80^{\circ}$ view was presented twice consecutively at study. The $80^{\circ}-80^{\circ}$ condition provided the control condition to ensure that two experiences with the same view of an object does not lead to better generalization to a novel view at test. We chose the $80^{\circ}-80^{\circ}$ condition rather than the $110^{\circ}-110^{\circ}$ condition because latencies to respond to the $80^{\circ}$ views in the study phases of Experiment 1 were numerically faster than latencies to respond to the $110^{\circ}$ views, suggesting that it may have been easier to perceive 3-D symmetry when objects were seen from the $80^{\circ}$ view. Thus, the $80^{\circ}-80^{\circ}$ condition was chosen in order to provide a more stringent comparison condition for examining whether or not experience with two different views allows for better generalization to novel views than does repeated experience with the same view.

Priming and improved recognition were expected in the $0^{\circ}-0^{\circ}$ condition because performance on both tasks is enhanced when the object is presented in the same viewpoint at study and test. Priming and improved recognition were also expected in the $80^{\circ}-110^{\circ}$ condition, if experience with two different views of an object leads to better generalization to novel views. Relatively less improvement was expected in priming or recognition in the $80^{\circ}-80^{\circ}$ condition because repeated experience with the same view of an object was not expected to facilitate responses to the $0^{\circ}$ test view. As in Experiment 1, these predictions were tested for symmetrical and asymmetrical objects. Because each object was presented twice in Experiment 2 , it was also expected that memory would be improved for asymmetrical objects in this experiment.

\section{Method}

Participants. Another group of 96 Boston College undergraduates participated in order to partially fulfill a course requirement.

Materials. The stimuli were exactly the same as those used in Experiment 1 .

Design. A 2 (test type: symmetry decision or recognition) $\times 2$ (study status: studied or nonstudied) $\times 3$ (study view: $0^{\circ}-0^{\circ}$, $80^{\circ}-110^{\circ}$, or $80^{\circ}-80^{\circ}$ ) mixed factorial design was used. Test type was manipulated between subjects, and study status and study view were manipulated within subjects. Forty-eight participants were given the symmetry decision task, and an additional 48 participants were assigned to the recognition task. For each task, 36 pairs of object views were presented in temporal succession at study, and 72 objects ( 36 studied and 36 nonstudied) were presented at test. Thus, at study, the participants would see 12 pairs consisting of $0^{\circ}-0^{\circ}$ views, 12 pairs of $80^{\circ}-110^{\circ}$ views, and 12 pairs of $80^{\circ}-80^{\circ}$ views in random order. Six study and test lists were constructed to achieve counterbalancing so that each object was presented in each condition equally often across subjects.

Procedure. The procedure was exactly the same as in Experiment 1 , except that the participants responded to each repetition of the object separately.

\section{Results and Discussion}

Symmetry decision task. Figure 4 represents latency (in milliseconds) for symmetrical and asymmetrical objects as a function of study condition $\left(0^{\circ}-0^{\circ}, 80^{\circ}-110^{\circ}\right.$, and $80^{\circ}-80^{\circ}$ conditions). As shown in Figure 4, statistically significant priming for responses to the $0^{\circ}$ test views of symmetrical objects was observed for the $0^{\circ}-0^{\circ}$ condition $\left[F(1,47)=22.42, M S_{\mathrm{e}}=111,725\right]$ and for the $80^{\circ}-110^{\circ}$ 

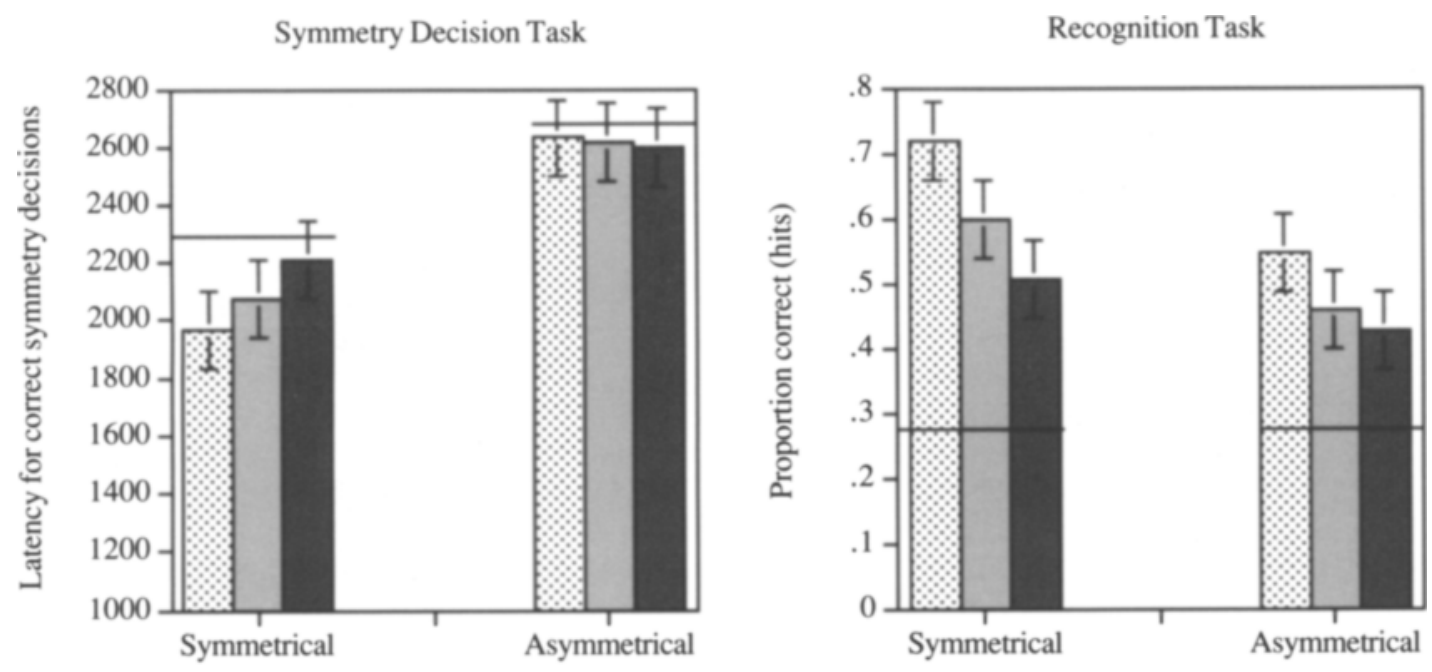

\begin{abstract}
Views at Study
$0^{\circ}-0^{\circ}$

$80^{\circ}-110^{\circ}$

$80^{\circ}-80^{\circ}$

Figure 4. The latency and accuracy results for the symmetry-asymmetry task and the recognition memory task of Experiment 2 for the $0^{\circ}-0^{\circ}, 80^{\circ}-110^{\circ}$, and $80^{\circ}-80^{\circ}$ conditions. Horizontal lines on the graphs indicate false-alarm rates for the recognition memory task and nonstudied base rates for the the symmetry decision task.
\end{abstract}

condition $\left[F(1,47)=7.81, M S_{e}=141,689\right]$, but not for the $80^{\circ}-80^{\circ}$ condition $(F<1)$. The advantage observed for the $80^{\circ}-110^{\circ}$ condition, suggests that seeing two different views of an object at study does improve generalization to a novel test view. Furthermore, the lack of priming for the $80^{\circ}-80^{\circ}$ condition, relative to the $80^{\circ}-110^{\circ}$ condition, suggests that the advantage observed for the $80^{\circ}-110^{\circ}$ condition is not attributable simply to repeated exposure to an object at study. Additional planned comparisons of the mean latencies in the different study conditions also suggested a statistically significant advantage for the $0^{\circ}-0^{\circ}$ and $80^{\circ}-110^{\circ}$ conditions relative to the $80^{\circ}-80^{\circ}$ condition $\left[F(1,47)=3.92, M S_{\mathrm{e}}=218,511\right]$.

Consistent with the findings in Experiment 1, analyses of the latency data for the asymmetrical objects produced no statistically significant priming for any of the three study conditions $(F \mathbf{s}<1)$. Similarly, there was no statistically significant difference between the combined $0^{\circ}-0^{\circ}$ and $80^{\circ}-110^{\circ}$ conditions versus the $80^{\circ}-80^{\circ}$ condition $(F<1)$. The lack of facilitation for asymmetrical objects in the condition that involved seeing exactly the same view of an object at study and test is consistent with the results of Experiment 1. In summary, the results with the asymmetrical objects suggest poor memory for these objects, despite the fact that the objects were presented twice to the participants in this experiment.

Recognition memory task. Figure 4 provides accuracy data for the recognition responses to symmetrical and asymmetrical objects presented at test for the three study conditions $\left(0^{\circ}-0^{\circ}, 80^{\circ}-110^{\circ}\right.$, and $80^{\circ}-80^{\circ}$ conditions $)$. For symmetrical objects, as illustrated in Figure 4 , recognition (hits - false alarms) was significantly above chance performance in the $0^{\circ}-0^{\circ}$ condition $[t(47)=12.14]$, the $80^{\circ}-110^{\circ}$ condition $[t(47)=9.80]$, and the $80^{\circ}-80^{\circ}$ condition $[t(47)=5.99]$, suggesting statistically significant recognition performance in each of the three study conditions. A planned comparison indicated a statistically significant advantage for mean accuracy in the $80^{\circ}-110^{\circ}$ condition over the $80^{\circ}-80^{\circ}$ condition $\left[F(1,47)=4.42, M S_{\mathrm{e}}=\right.$ $0.04]$, yielding further support for the prediction that experience with two different study views would result in increased generalization to novel test views. In addition, the difference between the $0^{\circ}-0^{\circ}$ and $80^{\circ}-110^{\circ}$ conditions was statistically significant $\left[F(1,47)=10.55, M S_{\mathrm{e}}=0.03\right]$, suggesting that exposure to two views did not result in the creation of view-independent representations. In summary, better generalization to a test view was observed when two different views of an object were presented at study (i.e., in the $80^{\circ}-110^{\circ}$ condition) relative to when objects were presented in a single view twice $\left(80^{\circ}-80^{\circ}\right)$.

Accuracy for asymmetrical objects was above chance in the $0^{\circ}-0^{\circ}$ condition $[t(47)=7.81]$, the $80^{\circ}-110^{\circ}$ condition $[t(47)=4.93]$, and the $80^{\circ}-80^{\circ}$ condition $[t(47)=$ 4.52]. However, there was no difference between the $80^{\circ}-$ $110^{\circ}$ and the $80^{\circ}-80^{\circ}$ conditions $(F<1)$. In addition, performance in the $0^{\circ}-0^{\circ}$ condition was better than that in the $80^{\circ}-110^{\circ}$ condition $\left[F(1,47)=4.62, M S_{\mathrm{e}}=0.04\right]$.

Two main conclusions can be drawn from the results of Experiment 2. First, experience with different views does appear to improve generalization to novel views of objects, at least for symmetrical objects. This generalization effect cannot be attributed to transfer from single 
views alone. Second, this generalization advantage does appear to occur across changes in viewpoint for basic-level discriminations of symmetrical objects (i.e., for discrimination of objects with unique part structures). The findings of Bulthoff and Edelman (1992) and Tarr (1995) therefore appear to be applicable to both within-category and basic-level identification tasks.

One factor that complicates the interpretation of Experiment 2 , however, is that the generalization was obtained with symmetrical, but not with asymmetrical, objects. This could reflect important processing differences between symmetrical and asymmetrical objects, as suggested by earlier researchers (Kersteen-Tucker, 1991), or it could simply reflect differences in the ability to remember asymmetrical objects. Asymmetrical objects tend to be, in general, more complex than symmetrical objects; therefore, floor effects for asymmetrical objects on the priming and recognition tasks might have masked any effects of generalization. We addressed this possibility in Experiment 3 by enhancing memory for asymmetrical objects and symmetrical objects.

\section{EXPERIMENT 3}

In Experiment 3, participants perceived the objects three times in the exact same conditions as in Experiment 2 (the $0^{\circ}-0^{\circ}, 80^{\circ}-110^{\circ}$, and $80^{\circ}-80^{\circ}$ conditions). Because each object was perceived three times in Experiment 2, this amounted to six repetitions of each object at study. We hypothesized that repetition should help in the representation of asymmetrical objects and should therefore result in improved memory performance in the $0^{\circ}-0^{\circ}$ condition on the symmetry decision and recognition memory tasks. The question of interest was whether the patterns of generalization obtained with symmetrical objects would also be obtained for asymmetrical objects.

\section{Method}

Participants. Another group of 84 Boston College undergraduates participated in order to fulfill a course requirement.

Materials. The stimuli were exactly the same as those used in Experiments 1 and 2.

Design. The design was exactly the same as in Experiment 2, except that the study lists were repeated in a different random order in three blocks. Forty-eight participants were given the symmetry decision task, and an additional 36 participants were assigned to the recognition task.

Procedure. The procedure was exactly the same as in Experiment 2 , except that the participants were asked to make symmetry decisions for objects appearing in each of three study blocks.

\section{Results and Discussion}

Symmetry decision task. Figure 5 represents latency (in milliseconds) for symmetrical and asymmetrical objects as a function of study condition $\left(0^{\circ}-0^{\circ}, 80^{\circ}-110^{\circ}\right.$, and $80^{\circ}-80^{\circ}$ conditions). As in Experiment 2 , statistically significant priming was observed for $0^{\circ}$ test views of symmetrical objects in the $0^{\circ}-0^{\circ}$ condition $[F(1,47)=63.37$, $\left.M S_{\mathrm{e}}=112,407\right]$ and the $80^{\circ}-110^{\circ}$ condition $[F(1,47)=$ 24.95, $\left.M S_{\mathrm{e}}=154,979\right]$. Unlike in Experiments 1 and 2, statistically significant priming was observed in the $80^{\circ}-80^{\circ}$ condition $\left[F(1,47)=12.69, M S_{\mathrm{e}}=105,646\right]$, presumably because of increased exposure to this view in this experiment relative to in the earlier experiments. When the objects are well learned or are highly familiar, local distinctive features of objects may be used to generalize to novel views (see Tarr, 1995, for this discussion).

Because statistically significant priming was observed in all three study conditions, the critical comparison for assessing the effect of view combination is the difference between the $80^{\circ}-110^{\circ}$ and $80^{\circ}-80^{\circ}$ conditions. This comparison indicated a statistically significant advantage for the $80^{\circ}-110^{\circ}$ condition $\left[F(1,47)=3.94, M S_{\mathrm{e}}=165,841\right]$, suggesting that seeing two different views of an object at study produces greater facilitation for the processing of a novel test view than does seeing one different view at study.

For asymmetrical objects, statistically significant facilitation was observed for the $0^{\circ}$ test views of objects in the $0^{\circ}-0^{\circ}$ condition $\left[F(1,47)=19.75, M S_{\mathrm{e}}=51,341\right]$, but not in the $80^{\circ}-110^{\circ}$ condition [by subjects, $F(1,47)=2.66$, $M S_{\mathrm{e}}=39,449, p<.11$; by items, $\left.F<1\right]$ or in the $80^{\circ}-80^{\circ}$ condition [by subjects, $F(1,47)=3.47, M S_{\mathrm{e}}=50,115$, $p<.07$; by items, $F<1]$. This pattern of results suggests that improved memory for asymmetrical objects was obtained after six repetitions of each object at study, but only when study and test views were identical. Other analyses confirmed this observation. Performance in the $0^{\circ}-0^{\circ}$ condition was significantly better than that in the $80^{\circ}-110^{\circ}$ condition $\left[F(1,47)=5.94, M S_{\mathrm{e}}=78,552\right]$, and, more importantly, no statistically significant difference was obtained between the $80^{\circ}-110^{\circ}$ and $80^{\circ}-80^{\circ}$ conditions $(F<1)$. Thus, the facilitation for asymmetrical objects appears to reflect viewpoint-sensitive representations, with little or no reliable generalization in the $80^{\circ}-110^{\circ}$ and $80^{\circ}-80^{\circ}$ conditions even after six exposures to each object at study.

Recognition memory task. Figure 5 provides accuracy data for the recognition responses to symmetrical and asymmetrical objects presented at test for the three study conditions $\left(0^{\circ}-0^{\circ}, 80^{\circ}-110^{\circ}\right.$, and $80^{\circ}-80^{\circ}$ conditions $)$. For symmetrical objects, as illustrated in Figure 5, analyses of the corrected accuracy data indicated that performance was significantly better than chance in the $0^{\circ}-0^{\circ}$ condition $[t(35)=28.21]$, the $80^{\circ}-110^{\circ}$ condition $[t(35)=$ $20.99]$, and the $80^{\circ}-80^{\circ}$ condition $[t(35)=13.07]$. A planned comparison between the $80^{\circ}-110^{\circ}$ condition and the $80^{\circ}-80^{\circ}$ condition indicated a statistically significant advantage for the $80^{\circ}-110^{\circ}$ condition $[F(1,35)=4.61$, $\left.M S_{\mathrm{e}}=0.02\right]$, suggesting better generalization to novel test views after seeing two different study views than after seeing one different study view. In summary, greater generalization to a novel test view was observed when two different views of an object were presented at study (i.e., in the $80^{\circ}-110^{\circ}$ condition) than when only one different view was presented at study (i.e., in the $80^{\circ}-80^{\circ}$ condition).

As predicted, increased exposure at study improved recognition memory for asymmetrical objects. Analyses of the accuracy data indicated that performance was significantly better than chance in the $0^{\circ}-0^{\circ}$ condition $[t(35)$ 

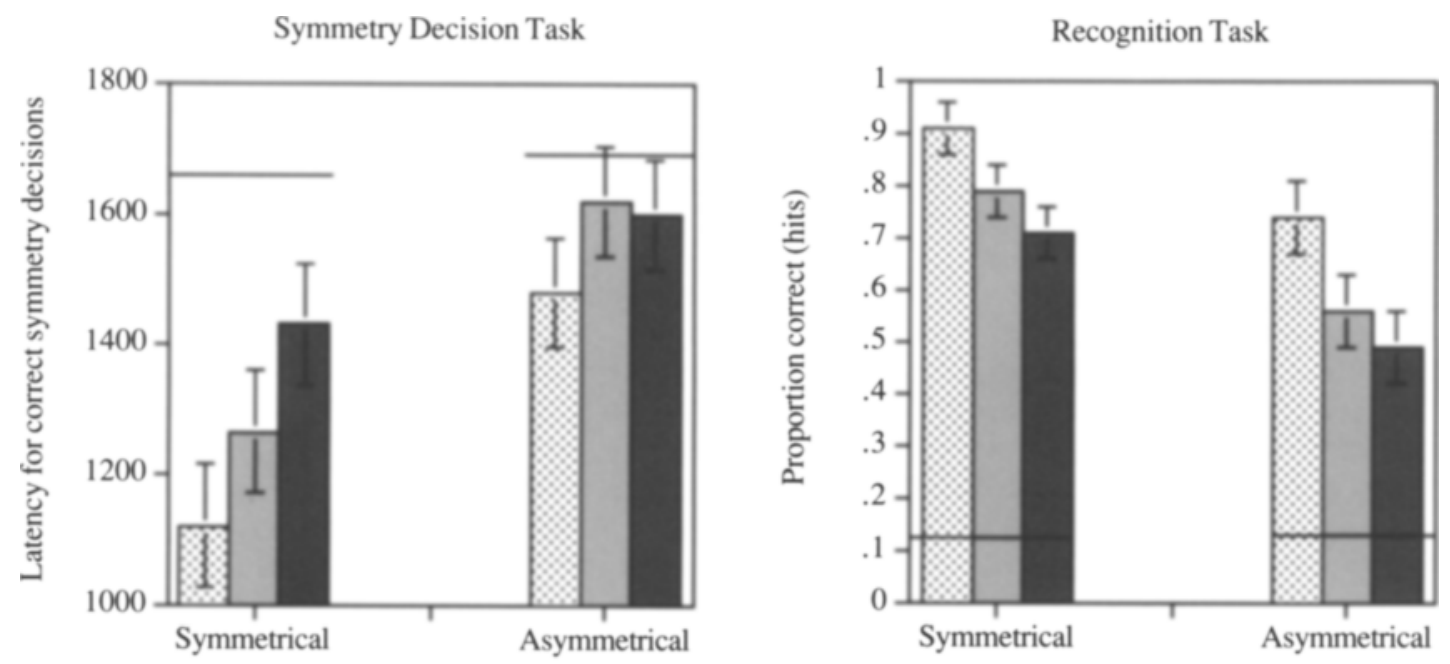

\begin{abstract}
Views at Study
$0^{\circ}-0^{\circ}$

$80^{\circ}-110^{\circ}$

$80^{\circ}-80^{\circ}$

Figure 5. The latency and accuracy results for the symmetry-asymmetry task and the recognition memory task of Experiment 3 for the $0^{\circ}-0^{\circ}, 80^{\circ}-110^{\circ}$, and $80^{\circ}-80^{\circ}$ conditions. Horizontal lines on the graphs indicate false-alarm rates for the recognition memory task and nonstudied base rates for the the symmetry decision task.
\end{abstract}

$=12.55]$, the $80^{\circ}-110^{\circ}$ condition $[t(35)=12.19]$, and the $80^{\circ}-80^{\circ}$ condition $[t(35)=6.55]$. Planned comparisons indicated a statistically significant advantage for the $0^{\circ}-0^{\circ}$ condition over the $80^{\circ}-110^{\circ}$ condition $[F(1,35)=$ $\left.17.05, M S_{\mathrm{e}}=0.03\right]$ and indicated no statistically significant difference between the $80^{\circ}-110^{\circ}$ and $80^{\circ}-80^{\circ}$ conditions $\left[F(1,35)=1.66, M S_{\mathrm{e}}=0.03, p<.21\right]$. These results suggest that, as in the symmetry decision task, recognition memory for asymmetrical objects is best when exactly the same views of objects are seen at study and test, and that little or no generalization is obtained for asymmetrical objects even after six repetitions of the object at study.

Two main conclusions can be drawn from the results of Experiment 3. First, the results of Experiment 3 replicate the findings of Experiment 2 and thus provide further support for the prediction that experience with more than one view would allow for better generalization to novel views of objects. This generalization effect cannot be attributed to generalization from single views alone. Second, this generalization appears to be restricted to symmetrical objects, and it does not appear to occur for asymmetrical objects in the present experiments. We return to this issue in the General Discussion section.

We next addressed whether the generalization advantage from view combination can occur on the basis of experience with different 2-D views of objects. In Experiments 2 and 3, because the study views were presented contiguously, it is possible that the generalization advantage occurred as a result of the extraction of 3-D information about the object. Because 3-D information was also presented to participants in prior research (Bulthoff \& Edelman, 1992; Tarr, 1995), it is unclear whether gen- eralization can indeed occur on the basis of experience with different 2-D views. We therefore addressed this issue in Experiment 4.

\section{EXPERIMENT 4}

In Experiment 4, we manipulated three conditions at study. The first two conditions $\left(0^{\circ}-0^{\circ}\right.$ condition and the sequential $80^{\circ}-110^{\circ}$ condition) were identical to those used in Experiments 2 and 3. The third condition used the same views as in the sequential $80^{\circ}-110^{\circ}$ condition, but the views were presented with a minimum lag of one intervening item, and, thus, this condition was called the spaced $80^{\circ}-110^{\circ}$ condition. The goal of Experiment 4 was to examine whether generalization to a novel view would occur without access to any 3-D information through apparent motion or through perception of the object in 3-D. The critical comparison was between the sequential $80^{\circ}-110^{\circ}$ condition and the spaced $80^{\circ}-110^{\circ}$ condition. The $0^{\circ}-0^{\circ}$ condition was included as a comparison condition, because it was expected to produce maximal priming.

\section{Method}

Participants. Another group of 96 Boston College undergraduates participated in order to partially fulfill a course requirement.

Materials. The stimuli were exactly the same as those used in Experiments 1-3.

Design. A 2 (test type: symmetry decision or recognition) $\times 2$ (study status: studied or nonstudied) $\times 3$ (study view: $0^{\circ}-0^{\circ}$, sequential $80^{\circ}-110^{\circ}$, or spaced $80^{\circ}-110^{\circ}$ ) mixed factorial design was used. Test type was manipulated between subjects, whereas study status and study view were manipulated within subjects. Forty-eight participants were given the symmetry decision task, and an additional 

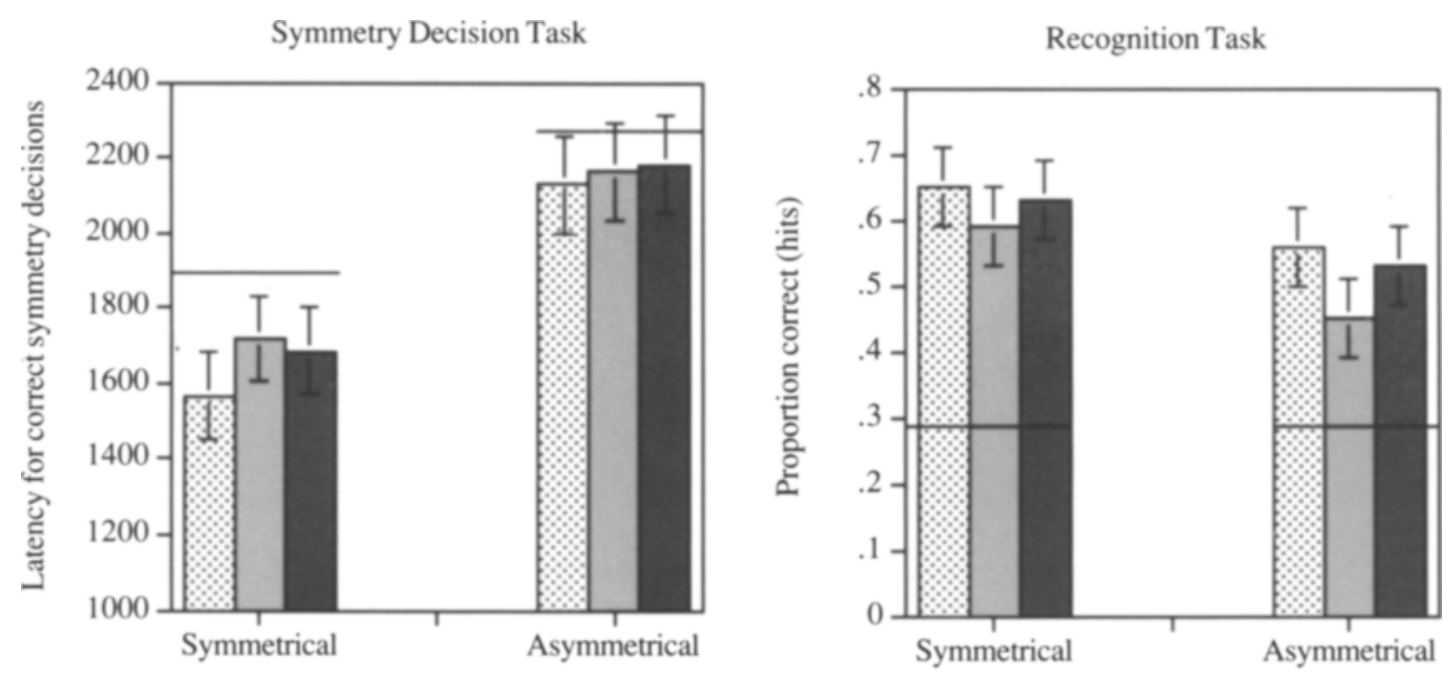

\section{Views at Study

Figure 6. The latency and accuracy results for the symmetry-asymmetry task and the recognition memory task of Experiment 4 for the $0^{\circ}-0^{\circ}$, sequential $80^{\circ}-110^{\circ}$, and spaced $80^{\circ}-110^{\circ}$ conditions. Horizontal lines on the graphs indicate false-alarm rates for the recognition memory task and nonstudied base rates for the symmetry decision task.

48 participants were assigned to the recognition task. All other aspects of the design were similar to Experiment 2, except that, in the spaced $80^{\circ}-110^{\circ}$ condition, the two views were separated by an average of 26 intervening views of other objects. Views were thus presented in a pseudorandom order at study, with intermixed trials from all three conditions.

Procedure. The procedure was exactly the same as in Experiment 2.

\section{Results and Discussion}

Symmetry decision task. Figure 6 represents latency (in milliseconds) for symmetrical and asymmetrical objects as a function of study condition $\left(0^{\circ}-0^{\circ}\right.$, sequential $80^{\circ}-110^{\circ}$, and spaced $80^{\circ}-110^{\circ}$ conditions). As expected, statistically significant priming was obtained in the $0^{\circ}-0^{\circ}$ condition $\left[F(1,47)=33.64, M S_{\mathrm{e}}=80,142\right]$ and the sequential $80^{\circ}-110^{\circ}$ condition $\left[F(1,47)=6.57, M S_{\mathrm{e}}=\right.$ $124,500]$. More importantly, statistically significant priming was also observed in the spaced $80^{\circ}-110^{\circ}$ condition $\left[F(1,47)=8.42, M S_{\mathrm{e}}=134,967\right]$, suggesting that generalization advantages after experience with more than one study view are not dependent on access to 3-D information from apparent motion. Additionally, the difference between the sequential $80^{\circ}-110^{\circ}$ condition and the spaced $80^{\circ}-110^{\circ}$ condition was not significant $(F<1)$, further suggesting that exposure to two different views at study allows for better generalization to novel test views regardless of whether 3-D information about the object is provided through apparent motion.

Taken together, the findings with symmetrical objects suggest that (1) perceiving two views in the sequential $80^{\circ}-110^{\circ}$ condition allows for improved generalization to a novel test view of the object, thus replicating the find- ings of Experiments 2 and 3, and, more importantly, (2) the two different study views do not have to be presented sequentially for generalization to occur. Thus, it appears that the observed generalization advantage is not dependent on the extraction of 3-D information due to kinetic depth effects, as in Bulthoff and Edelman (1992), or to seeing actual 3-D objects, as in Tarr (1995).

Consistent with the findings of Experiments 1 and 2, no statistically significant priming was obtained for asymmetrical objects. The only effect that approached significance was the priming effect for the $0^{\circ}-0^{\circ}$ condition in the analysis by subject variability $\left[F(1,47)=3.31, M S_{\mathrm{e}}=\right.$ $140,537, p<.08]$, but not by item variability $[F(1,35)=$ $\left.1.79, M S_{\mathrm{e}}=158,636, p<.19\right]$. These results replicate the poor memory for asymmetrical objects observed in Experiments 1 and 2, after two exposures to the studied object.

Recognition memory task. Figure 6 provides accuracy data for the recognition responses to symmetrical and asymmetrical objects presented at test for the three study conditions $\left(0^{\circ}-0^{\circ}\right.$, sequential $80^{\circ}-110^{\circ}$, and spaced $80^{\circ}-110^{\circ}$ conditions). As can be seen in Figure 6, mean accuracy for symmetrical objects was significantly above chance for the $0^{\circ}-0^{\circ}$ condition $[t(47)=8.36]$, the sequential $80^{\circ}-110^{\circ}$ condition $[t(47)=8.89]$, and the spaced $80^{\circ}-110^{\circ}$ condition $[t(47)=8.47]$. Unlike in Experiments $1-3$, no statistically significant difference was observed between either the $0^{\circ}-0^{\circ}$ condition and the sequential $80^{\circ}-110^{\circ}$ condition $\left[F(1,47)=1.93, M S_{\mathrm{e}}=0.04\right.$, $p<.17$ ], or between the $0^{\circ}-0^{\circ}$ condition and the spaced $80^{\circ}-110^{\circ}$ condition $(F<1)$, suggesting almost complete generalization from exposure to two views. More im- 
portantly, a planned comparison yielded no difference between the sequential $80^{\circ}-110^{\circ}$ and spaced $80^{\circ}-110^{\circ}$ conditions $(F \leq 1)$, suggesting that generalization to novel test views on a recognition memory task is improved by exposure to two different study views, regardless of whether or not it is possible to extract 3-D information from the two study views. In sum, these findings are consistent with the prediction that experience with more than one view results in better generalization to novel views and that this generalization advantage is not dependent on the extraction of 3-D information.

As in Experiments 1-3, recognition memory performance for asymmetrical objects was significantly above chance in the $0^{\circ}-0^{\circ}$ condition $[t(47)=8.08]$, the sequential $80^{\circ}-110^{\circ}$ condition $[t(47)=4.34]$, and the spaced $80^{\circ}-110^{\circ}$ condition $[t(47)=6.51]$. Planned comparisons indicated an advantage for the $0^{\circ}-0^{\circ}$ condition over the sequential $80^{\circ}-110^{\circ}$ condition $[F(1,47)=9.49$, $\left.M S_{\mathrm{e}}=0.03\right]$ but indicated no advantage for the $0^{\circ}-0^{\circ}$ condition over the spaced $80^{\circ}-110^{\circ}$ condition $(F<1)$. The difference between the sequential $80^{\circ}-110^{\circ}$ and spaced $80^{\circ}-110^{\circ}$ conditions was marginally significant $[F(1,47)=$ $\left.3.87, M S_{\mathrm{e}}=0.05, p<.06\right]$ but was in a direction opposite to the predicted pattern. Specifically, performance in the spaced $80^{\circ}-110^{\circ}$ condition was better than performance in the sequential $80^{\circ}-110^{\circ}$ condition. We have no explanation for this latter finding, except that we note that this finding is probably not replicable. In Experiment 3 , when the $80^{\circ}-110^{\circ}$ views were presented sequentially but were also spaced in three separate repetitions, recognition memory for the $0^{\circ}-0^{\circ}$ condition was better than memory for the $80^{\circ}-110^{\circ}$ condition; however, in $\mathrm{Ex}$ periment 4 , the two conditions were almost equivalent. We therefore conclude that generalization is difficult for asymmetrical objects relative to symmetrical objects.

\section{GENERAL DISCUSSION}

Three main findings emerged from the present set of experiments. First, performance on both measures of memory was sensitive to the studied viewpoint of a novel object. Specifically, when the participants experienced only one view of an object at study, reduced facilitation was obtained for novel test views. This was true for both symmetrical and asymmetrical objects. Together with the findings of prior studies, the findings of Experiment 1 support the idea that objects are represented in specific experienced views in memory.

Second, on both measures of memory, improved generalization to extrapolated views of symmetrical objects was observed after exposure to two 2-D views of each object at study. Importantly, this generalization advantage (1) occurred on tasks that required basic-level object discrimination and (2) did not appear to depend on access to 3-D information about the objects. Consider now an alternative explanation of our findings. One might argue from a part-based account (Biederman \& Gerhardstein, 1993) that two views of a novel object simply allow better specification of a part-based representation, perhaps because the two views jointly specify a part structure that is not visible from a single view alone, and this part structure is most similar to the test view. We had explicitly tried to rule out this possibility by choosing three views that appeared to share the same parts or that did not include obvious part occlusions. Yet, as discussed earlier, in Experiment 1, we obtained little or no evidence of transfer from either of these two views to the test view despite visibility of the same parts. Furthermore, in the recognition memory task of the present experiments, generalization obtained from the perception of two different views $\left(80^{\circ}-110^{\circ}\right)$ was still not equivalent to that obtained from perception of the tested view $\left(0^{\circ}-0^{\circ}\right)$. In other words, exposure to two views did not result in view-independent performance. These results suggest that exposure to two views simply does not result in a part-based representation of the form proposed by Biederman and Gerhardstein (1993).

Third, memory for asymmetrical objects was consistently poor relative to memory for symmetrical objects a result that is consistent with earlier findings reported by Kersteen-Tucker (1991). Furthermore, under conditions in which performance was above chance for asymmetrical objects, little or no advantage was observed from exposure to two different study views of an object $\left(80^{\circ}-110^{\circ}\right)$ relative to exposure to a single view twice $\left(80^{\circ}-80^{\circ}\right)$. This result is different from the consistent generalization advantage observed for symmetrical objects in the $80^{\circ}-110^{\circ}$ condition. We hypothesize that this selective difficulty for asymmetrical objects is a result of their relatively unpredictable structure. Specifically, because the image structure of asymmetrical objects is more difficult to predict from a different view relative to symmetrical objects, generalization may be a more difficult process for these objects. This interpretation is consistent with psychophysical data of Vetter, Poggio, and Bulthoff (1994), who reported better generalization to novel "virtual views" for symmetrical objects than for asymmetrical objects.

We should point out that our results do not indicate that improved generalization is impossible for asymmetrical objects. Indeed, under conditions of intensive training with small sets of asymmetrical objects, generalization to novel views through view combination has been documented by Bulthoff and Edelman (1992) and by Tarr (1995). Together with these prior results, our data suggest that generalization to novel views is difficult, but not impossible, for asymmetrical objects. In conclusion, the present results suggest that (1) view combination allows generalization to novel views in within-category discrimination and in basic-level identification, (2) view combination can occur strictly on the basis of 2-D views of objects, and (3) view combination is more easily observed for symmetrical objects than for asymmetrical objects.

\section{REFERENCES}

BARTRAM, D. J. (1974). The role of visual and semantic codes in object naming. Cognitive Psychology, 6, 325-356.

Biederman, I,, \& Gerhardstein, P. C. (1993). Recognizing depth- 
rotated objects: Evidence and conditions for three-dimensional viewpoint invariance. Journal of Experimental Psychology: Human Perception \& Performance, 19, 1162-1182.

BulthoFf, H. H., \& Edelman, S. (1990, May). Recognizing objects from novel viewpoints. Paper presented at the annual meeting of the Association for Research in Vision and Ophthalmology, Sarasota, FL.

Bulthoff, H. H., \& Edelman, S. (1992). Psychophysical support for a two-dimensional view interpolation theory of object recognition. Proceedings of the National Academy of Sciences, 89, 60-64.

Bulthoff, H. H., Edelman, S., \& Sklar, E. (1991, May). Mapping the generalization space in object recognition. Paper presented at the annual meeting of the Association for Research in Vision and Ophthalmology, Sarasota, FL.

Edelman, S., \& Bulthoff, H. H. (1992). Orientation dependence in the recognition of familiar and novel views of three-dimensional objects. Vision Research, 32, 2385-2400.

HUMPHREY, G. K., \& KHAN, S. (1992). Recognizing novel views of threedimensional objects. Canadian Journal of Psychology, 46, 170-190.

KERSTEEN-TUCKER, Z. (1991). Long-term repetition priming with symmetrical polygons and words. Memory \& Cognition, 19, 37-43.

LoFTus, G. R., \& MASSON, M. E. J. (1994). Using confidence intervals in within-subject designs. Psychonomic Bulletin \& Review, 1, 476-490.

RATCLIFF, R., \& MCKoON, G. (1995). Bias in the priming of object decisions. Journal of Experimental Psychology: Learning, Memory, \& Cognition, 21, 754-767.

Rock, I., \& DiVITA, J. (1987). A case of viewer-centered object perception. Cognitive Psychology, 19, 280-293.

Rock, I., DiVita, J., \& Barbeito, R. (1981). The effect on form per- ception of change of orientation in the third dimension. Cognitive Psychology, 19, 280-293.

SRINIVAS, K. (1993). Perceptual specificity in nonverbal priming. Journal of Experimental Psychology: Learning, Memory, \& Cognition, 19, 582-602.

SRINIVAS, K. (1995). Representation of rotated objects in explicit and implicit memory. Journal of Experimental Psychology: Learning, Memory, \& Cognition, 21, 1019-1036.

TARR, M. J. (1995). Rotating objects to recognize them: A case study on the role of viewpoint dependency in the recognition of threedimensional objects. Psychonomic Bulletin \& Review, 2, 55-82.

Tarr, M. J., Hayward, W. G., Gauthier, I., \& Williams, P. (1994, November). Geon recognition is viewpoint dependent. Paper presented at the annual meeting of the Psychonomic Society, St. Louis.

Vetter, T, Poggio, T., \& Bulthoff, H. H. (1994). The importance of symmetry and virtual views in three-dimensional object recognition. Current Biology, 4, 18-23.

\section{NOTE}

1. The $95 \%$ confidence intervals displayed in the figure were calculated using the within-subjects $M S_{\mathrm{e}}$ for the effects of study view rather than the effects of study status. The confidence interval for the $110^{\circ}$ view, therefore, cannot be used to estimate the significance of the priming effect in that condition.

(Manuscript received December 30, 1996; revision accepted for publication May 28, 1997.) 